\title{
AUTOCUIDADO ENTRE MULHERES COM FATOR FAMILIAR DE CÂNCER DE MAMA
}

\author{
Maraísa Pimenta Vilela¹, Clícia Valim Côrtes Gradim², Lana Ermelinda da Silva dos Santos³, Eliza Maria Rezende Dázio ${ }^{4}$
}

\begin{abstract}
RESUMO: Trata-se de um estudo descritivo prospectivo que objetivou identificar, entre filhas e irmãs de mulheres com câncer de mama, o conhecimento do risco para essa neoplasia e quais práticas de autocuidado elas adotaram. Os dados foram coletados por meio da aplicação de um formulário em irmãs ou filhas de mulheres participantes do Projeto Mulher com Câncer de Mama, no período de 2004 a 2007. Das 20 mulheres entrevistadas 14 eram filhas, 5 irmãs e uma é filha e irmã, com idade média de 35 anos, alfabetizadas, sendo que 75\% relataram ter recebido alguma orientação sobre os aspectos preventivos; 65\% realizam o auto-exame das mamas e o exame clínico, 25\% fizeram mamografia e ultrassonografia de base. O profissional de saúde deve saber abordar a mulher com histórico de neoplasia mamária para estimular estratégias que possibilitem a adoção de práticas de autocuidado.
\end{abstract}

PALAVRAS-CHAVE: Neoplasias da mama; Autocuidado; Grupos de risco; Enfermagem.

\section{SELF CARE AMONG WOMEN WITH FAMILY FACTOR OF BREAST CANCER}

\begin{abstract}
This is a prospective descriptive study that aimed to identify, among daughters and sisters of women with breast cancer, the knowledge of the risk for this cancer and which self care practices they have adopted. Data were collected by the application of a formulary in sisters or daughters of the participant women from the Woman with Breast Cancer Project, in the period of 2004-2007. Of the 20 women interviewed, 14 were daughters, 5 sisters and one was daugther and sister of women with breast cancer, the average age was of 35 years, alphabetized, 75\% of them reported they had received some orientation about preventive aspects; $65 \%$ of them carry out both the self-examination and the clinical examination of the breasts; 25\% had made mammography and base ultrasonography. The health professional must know how to approach the woman with family history of mammary neoplasm to stimulate strategies that might allow the adoption of self-care practices.
\end{abstract}

KEYWORDS: Breast cancer; Self-care; Risk group; Nursing.

\section{AUTOCUIDADO ENTRE MUJERES CON FACTOR FAMILIAR DE CÁNCER DE PECHO}

RESUMEN: Se trata de un estudio descriptivo prospectivo que con el objetivo de identificar, entre hijas y hermanas de mujeres con cáncer de pecho, el conocimiento del riesgo para esta neoplasia y qué prácticas de autocuidado ellas adoptaron. Los datos fueron recogidos por medio de la aplicación de un formulario en hermanas o hijas de mujeres participantes del Proyecto Mujer con Cáncer de Pecho, en el período de 2004 a 2007. De 20 mujeres entrevistadas, 14 eran hijas, 5 hermanas y una es hija y hermana, con edad promedio de 35 años, alfabetizadas, siendo que 75\% relató haber recibido alguna orientación sobre los aspectos preventivos; 65\% realiza autoexamen de los pechos y el examen clínico, 25\% había hecho mamografía y ultrasonografía de base. El profesional de salud debe saber acercarse a la mujer con histórico de neoplasia mamaria para estimular estrategias que posibiliten la adopción de prácticas de autocuidado.

PALABRAS CLAVE: Neoplasias del pecho; Autocuidado; Grupos de riesgo; Enfermería.

${ }^{1}$ Discente do Curso de Graduação em Enfermagem da Universidade Federal de Alfenas-Unifal-MG.

${ }^{2}$ Enfermeira. Doutora. Professora Associada do Departamento de Enfermagem da Unifal-MG. Coordenadora do Projeto Mulheres com Câncer de Mama-Mucama.

${ }^{3}$ Enfermeira. Pós doutora. Professora Associada do Departamento de Enfermagem da Unifal-MG.

${ }^{4}$ Enfermeira. Doutora. Professora Adjunta do Departamento de Enfermagem da Unifal-MG.

Autor correspondente:

Maraísa Pimenta Vilela

Rua Pará, 1155 - 14401-410 - Franca-SP

Recebido: 18/12/08

E-mail:maraisa_vilela@yahoo.com.br

Aprovado: 01/06/09

Cogitare Enferm 2009 Abr/Jun; 14(2):254-60 


\section{INTRODUÇÃO}

O câncer é o nome dado a mais de cem patologias nas quais há o desenvolvimento desordenado das células, levando à formação de um tumor que afeta tecidos e órgãos. Manifesta-se por causas internas e externas, sendo que somente $10 \%$ estão relacionados a fatores de pré-disposição familiar ou hereditário. Dentre os vários tipos de câncer, o de mama tem sido uma preocupação na saúde pública, devido ao aumento progressivo de sua incidência, sendo a maior causa de mortalidade da mulher brasileira ${ }^{(1)}$.

No Brasil, o diagnóstico tardio tem sido o responsável por tratamentos agressivos como a mastectomia, levando a alteração da auto-imagem e a problemas psicológicos, além do alto custo para o sistema de saúde do país. Infelizmente ações de promoção e prevenção são pouco discutidas com as mulheres pelos profissionais de saúde, tendo como consequência a não adoção de hábitos de saúde e a ausência do autocuidado. Fatores de risco não são discutidos com as mulheres no sentido de elas se conscientizarem da importância de se cuidarem e de contribuírem com a sua saúde, visto que a história familiar de câncer de mama não pode ser alterada.

Uma das propostas de intervenção para o controle do câncer de mama está fundamentada no mapeamento do risco a que as mulheres estão expostas e no planejamento e na implementação de ações que visem à detecção de tumores os menores possíveis. Nesse sentido, o mapeamento do risco relacionado à história familiar tem sido considerado como um fator de grande importância uma vez que, entre os vários aspectos relacionados com o risco de desenvolvimento do câncer de mama, o fator familiar é o mais aceito na comunidade científica ${ }^{(2)}$. Mulheres com mãe e irmã com câncer de mama apresentam duas a três vezes mais risco, e, se ambas, mãe e irmã, tiverem a doença, o risco aumenta ainda mais, especialmente se a doença delas tiver ocorrido antes da menopausa ${ }^{(3)}$.

Entretanto, apesar de haver o conhecimento de que o câncer tem uma predisposição familiar, a atuação na prevenção e promoção à saúde com filhas e irmãs dessas mulheres é tímida, quando não inexistente. Os fatores de risco são situações que aumentam a probabilidade do desenvolvimento da patologia e podem ser modificados com mudanças de hábitos de vida ${ }^{(4)}$.

Os fatores de risco para ao câncer de mama são: tabagismo, etilismo, uso de anticoncepcionais por longo tempo, sedentarismo, alimentação gordurosa, estresse, nuliparidade, ocorrência da primeira gravidez após os 30 anos, anormalidades anteriores da mama e pré-disposição familiar ${ }^{(2)}$. Cientes disso, os profissionais de saúde devem estimular as mulheres à prática de hábitos saudáveis e à mudança de comportamentos, pois ter uma boa alimentação, praticar exercícios, evitar o uso de álcool e tabaco e realizar exames periodicamente são ações de promoção de saúde.

Nesse sentido, este estudo tem como objetivo identificar, entre filhas e irmãs de mulheres com câncer de mama, seu conhecimento acerca dos riscos para essa neoplasia e investigar quais práticas de autocuidado elas adotam para o controle da mesma.

\section{METODOLOGIA}

O estudo aplicado é descritivo prospectivo, do tipo coorte de uma determinada população especial (irmãs ou filhas de mulheres com câncer de mama). "Os estudos de coorte têm início ao se colocar em foco uma variável cuja contribuição como fator de risco para determinada doença se deseja conhecer, avaliar ou confirmar ${ }^{(5)}$ ".

A coleta de dados foi realizada nos anos de 2004 a 2007 com familiares de mulheres participantes do Projeto Mulher com Câncer de Mama-Mucama da Universidade Federal de Alfenas-Unifal, por meio da aplicação de um formulário.

A amostra ficou constituída por 20 mulheres que se enquadraram nos fatores de inclusão de: ter mais de 18 anos, ter irmã ou mãe com diagnóstico de câncer de mama e que concordaram em participar da pesquisa.

Após a concordância da participação, foi marcado local e horário prévio para a aplicação do formulário, sendo que 9 foram realizadas nas dependências do projeto Mucama e 11 no domicílio. O instrumento permitiu levantar dados socioeconômicos, conhecimento sobre o câncer de mama, recebimento de orientações sobre o mesmo e práticas de autocuidado relacionadas à prevenção do câncer de mama.

Os dados foram coletados após aprovação do projeto pelo Comitê de Ética de Pesquisa da UnifalMG, processo n. 23087.001926-2003. Os dados foram organizados, analisados por meio da estatística simples e apresentados em tabelas e gráficos.

\section{RESULTADOS E DISCUSSÃO}

Quanto às características socioeconômicas, as participantes encontram-se na faixa etária entre 18 e 52 anos, sendo que 9 (45\%) têm idade superior a 38 anos. Essa faixa etária também foi encontrada em um 
estudo realizado no Ceará sobre fatores de risco para o câncer de mama, no qual se observa que a maior parte da amostra é composta por mulheres com idade superior aos 38 anos $^{(6)}$. Esse fator requer uma atenção especial nas medidas de detecção precoce do câncer de mama, pois quanto mais avançada for a idade, maior o risco de desenvolver essa patologia.

No Brasil tem-se observado uma mudança na taxa de incidência do câncer de mama, a qual aumenta rapidamente até os 50 anos, e posteriormente o mesmo se dá de forma mais lenta ${ }^{(1)}$.

Quanto ao estado civil, os resultados foram equivalentes, pois 7 (35\%) mulheres são casadas, 7 (35\%) solteiras e 6 (30\%) separadas; $60 \%$ relataram ser prestadoras de serviço; quanto à escolaridade, todas são alfabetizadas e com relação à religião, a maioria das mulheres (80\%) são católicas. Quanto à renda familiar, 85\% das entrevistadas afirmaram possuir renda entre 1 a 6 salários mínimos. O câncer de mama tem maior incidência em países desenvolvidos e em famílias de classes sociais mais altas ${ }^{(7)}$. No entanto, na realidade do município, essa patologia atinge todas as mulheres, indiscriminadamente, independentemente de situação social ou renda.

Alfenas-MG encontra-se entre os 14 municípios do estado de Minas Gerais com maior índice de mortalidade para o câncer de mama ${ }^{(8)}$. Essa estimativa reforça a importância e a prioridade que se deve dar às ações de prevenção e controle, além de ser um alerta principalmente para as mulheres que possuem como fator de risco o câncer de mama intrafamiliar.

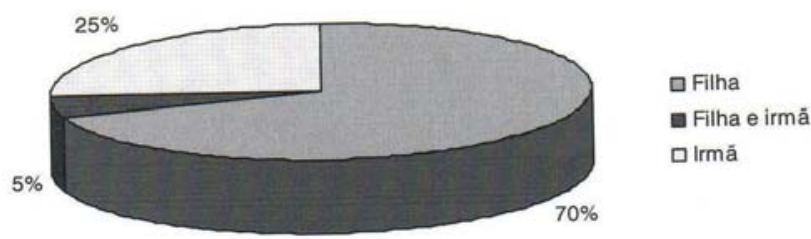

Figura 1 - Grau de parentesco das mulheres com fator familiar para câncer de mama. Alfenas-MG, 2007

A Figura 1 representa o grau de parentesco entre o grupo estudado e a relação com o familiar para câncer de mama. Essa relação é um fator de risco bem conhecido, por isso essas mulheres devem ser instruídas a terem ações de promoção e proteção para esse fator, apesar de estudos mostrarem que apenas $10 \%$ das mulheres diagnosticadas com câncer de mama têm uma história familiar positiva ${ }^{(7)}$.

O risco de câncer de mama é duas vezes maior quando há história familiar em mãe ou irmã antes dos 40 anos, e continua elevado mesmo em mulheres cujo diagnóstico em mãe foi após os 70 anos de idade. Neste estudo, 35\% das mães ou irmãs das entrevistadas foram acometidas pela doença nas faixas etárias de risco; destas, 3 (15\%) entre 25 e 40 anos e 4, (20\%) acima dos 70 anos; porém, 65\% tiveram o câncer na faixa etária de maior incidência, entre 45 e 65 anos $^{(9)}$. Esses resultados reforçam a importância do conhecimento das práticas de autocuidado, e de os profissionais de saúde reforçarem sobre a relevância de possuir hábitos saudáveis para minimizar os fatores de risco.

Para avaliar o conhecimento e a relação dessas mulheres frente aos fatores de risco para o câncer de mama, questionou-se sobre a dieta, sendo que $50 \%$ delas afirmaram consumir alimentos ricos em gorduras. A ingestão excessiva de gorduras de origem animal, polissaturadas, associa-se de forma positiva ao risco de desenvolvimento de neoplasia mamária, pois propicia o metabolismo intestinal dos esteróides biliares em estrogênio, sob ação de bactérias ${ }^{(10)}$. É importante lembrar que no Estado de Minas Gerais o consumo de gordura de porco é ainda uma tradição na culinária, sendo o papel dos profissionais alertar sobre a importância de se ter uma dieta saudável, com grande quantidade de frutas, verduras e legumes ${ }^{(11)}$.

Associado a esses hábitos alimentares, é fundamental que essas mulheres se preocupem em manter um peso adequado, pois o tecido adiposo apresenta inusitada capacidade de converter androstenediona em estrogênica, o que poderia desencadear, em longo prazo, uma proliferação tanto do tecido mamário quanto do endométrio ${ }^{(10)}$.

Com relação a hábitos etílicos e câncer de mama, o Risco Relativo-RR é de 2,45 superior de desenvolver a doença se comparado ao daquelas que nunca consomem álcool. Quanto ao tabagismo, o RR de 2,4 vezes maior quando comparado ao daquelas não fumantes ${ }^{(13)}$. $\mathrm{Na}$ amostra, 6 (30\%) das mulheres relataram ser tabagistas e 1 (5\%) afirmou consumir bebida alcoólica diariamente. É importante que essas mulheres sejam estimuladas a reduzir esses fatores de risco, pois o não uso do cigarro e da bebida alcoólica, a prática regular de atividade física e uma dieta saudável podem reduzir em até $50 \%$ a incidência do câncer de mama ${ }^{(11)}$.

Quando questionadas com relação ao contraceptivo hormonal oral-CHO, 70\% das mulheres fazem uso, sendo que 78,57\% em um período inferior a cinco anos, e 21,43\%, em um período superior a 12 anos. O CHO não aumenta a incidência de câncer de mama quando usado por um período igual ou inferior a dez anos. No entanto, quando o período aumenta de dez a 15, o CHO oferece pequeno risco, e, após os 15 
anos, ocorre aumento real do risco, principalmente quando iniciado em idade precoce, especialmente antes da primeira gestação a termo ${ }^{(13)}$, mas isso é controverso, pois outros estudos não associam o uso de contraceptivos orais com o aumento do risco para o câncer de mama ${ }^{(1)}$.

As entrevistadas relataram nunca terem feito uso da terapia de reposição hormonal, o que se deve ao fato de apenas uma mulher estar na fase da menopausa.

A Tabela 1 se refere à distribuição percentual das mulheres segundo a idade da menarca, da primeira gestação, da menopausa e à nuliparidade, os quais podem ser fatores de risco ou proteção para o câncer de mama. Apenas 2 (10\%) mulheres apresentaram menarca dentro da faixa etária de risco, 12 (92,3\%) tiveram a primeira gestação dentro da faixa etária esperada, entre 18 e 29 anos. Porém, 7 (35\%) mulheres são nulíparas e, destas, 3 (42,86\%) possuem idade acima de 35 anos, sendo importante os profissionais alertarem que esse fator aumenta o risco de desenvolver a doença.

Tabela 1 - Distribuição das mulheres segundo a idade da menarca, da primeira gestação, da menopausa e à nuliparidade. Alfenas-MG. 2007

\begin{tabular}{ccccccccccc}
\hline $\begin{array}{c}\text { Idade da } \\
\text { Menarca }\end{array}$ & $\begin{array}{c}\text { N. } \\
\text { Absoluto }\end{array}$ & $\%$ & $\begin{array}{c}\text { Idade da } \\
\text { primeira } \\
\text { gestação }\end{array}$ & $\begin{array}{c}\text { N. } \\
\text { Absoluto }\end{array}$ & $\%$ & Nulíparas & $\begin{array}{c}\text { N. } \\
\text { Absoluto }\end{array}$ & $\begin{array}{c}\text { Idade da } \\
\text { Menopausa }\end{array}$ & $\begin{array}{c}\text { N. } \\
\text { Absoluto }\end{array}$ & $\%$ \\
\hline 11 & 2 & 10 & $14-17$ & 1 & 7,69 & 7 & 35 & 45 & 1 & 5 \\
12 & 9 & 45 & $18-21$ & 7 & 53,85 & & & & & \\
13 & 3 & 15 & $22-25$ & 2 & 15,38 & & & & \\
14 & 5 & 25 & $26-29$ & 7 & 23,08 & & & & & \\
15 & 1 & 5 & & & & & & & & \\
\hline
\end{tabular}

A menarca precoce, anterior aos 12 anos de idade, a menopausa tardia, após os 50 anos, e a idade avançada no primeiro parto, maior que 30 anos, estão intimamente relacionadas a um aumento no risco relativo de ter câncer de mama, uma vez que nestas situações as mulheres ficam expostas a estrógenos por maior período de tempo. O risco de desenvolvimento de câncer de mama em mulheres que tiveram seu primeiro filho após os 30 anos é aproximadamente o dobro daquelas que o fizeram antes dos $20^{(7)}$.

Em relação ao aleitamento materno, um fator protetor, das 13 mulheres que tiveram filhos, 11 $(84,62 \%)$ amamentaram por um período superior a seis meses. Um estudo realizado na Grã-Bretanha revelou que amamentar por pelo menos um ano reduz os riscos de desenvolver o câncer de mama em 48\%, sendo que esses 12 meses de amamentação não precisam ser contínuos, ou seja, amamentar dois bebês durante seis meses produz o mesmo efeito. Quanto maior e mais precoce for o aleitamento, maior será este efeito "protetor", pois a amamentação induz o amadurecimento das glândulas mamárias, tornando as células mais "estáveis" e menos suscetíveis ao desenvolvimento do câncer. Indiretamente, ao amamentar, essas mulheres minimizaram o seu fator de $\operatorname{risco}^{(14)}$.

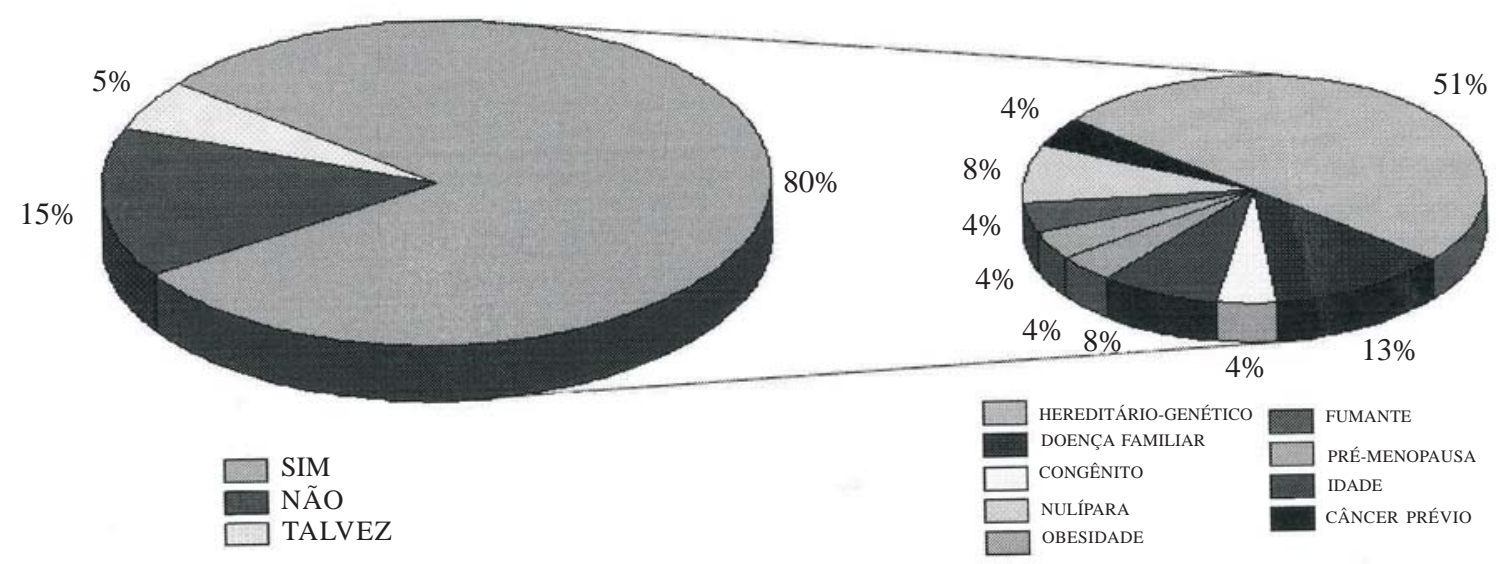

Figura 2 - Relação do aparecimento do câncer de mama com o fato da mãe ou irmã ter tido e causas. Alfenas-MG, 2007 
Como se observa na Figura 2, quando questionadas se acreditam que existe uma maior probabilidade de terem o câncer de mama pelo fato de a mãe ou irmã ter tido a patologia, a maioria das entrevistadas (80\%) respondeu positivamente, e indicaram vários fatores como propiciadores. Porém o fator genético-hereditário foi apontado como a principal causa, no entanto, é importante ressaltar que, apesar de o câncer de mama ter uma pré-disposição familiar, ele não é genético ${ }^{(3)}$.

Porém, essa afirmativa tem sido questionada, pois atualmente três genes têm sido apontados como principais no desenvolvimento do câncer hereditário de mama - o BRCA1, BRCA2 e o TP53. No entanto, as alterações encontradas nesses genes são responsáveis por aproximadamente $5 \%$ de todos os casos. Dentre os genes envolvidos nas etapas de desenvolvimento do câncer esporádico de mama, está o TP53, que é o gene que se apresenta com mais mutação na doença ${ }^{(15)}$. Esse dado reforça a importância ainda maior da orientação a essas mulheres que possuem história familiar de câncer de mama, sobre as práticas de autocuidado e sobre a minimização dos fatores de risco.

Questionou-se se essas mulheres receberam algum tipo de orientação sobre ações de promoção e prevenção do câncer de mama, sendo que 15 (75\%) afirmaram terem sido orientadas. As pessoas responsáveis por essa orientação foram o médico (73,3\%), as próprias mães ou irmãs (13,33\%) e duas não se lembram quem as orientou. Essa orientação foi individual (33,33\%) ou em consultas em que eram acompanhantes (12,5\%). O médico, assim como todos os profissionais de saúde, é um importante agente disseminador de informações sobre câncer de mama, por isso é essencial que se mantenham familiarizados com as recomendações e métodos disponíveis para prevenção do câncer de mama, para estimularem as mulheres ao autocuidado ${ }^{(16)}$.

Dos meios de comunicação, a mídia/TV $(20,83 \%)$ foi a mais citada para obtenção de informação quanto à prevenção do câncer de mama; dados coincidentes com outros estudos referem que “a TV tem um grande poder de penetração em todas as camadas sociais, sugerindo que as campanhas de combate ao câncer de mama devem necessariamente usar este instrumento de comunicação como elemento veiculador"(16).

O controle do câncer de mama deve priorizar a prevenção e a detecção precoce. A prevenção não deve focalizar apenas os fatores de risco do câncer de mama, mas também os de proteção e ações de prevenção, sendo que as formas mais eficazes para a detecção precoce do câncer de mama são o autoexame das mamas, o exame clínico, a mamografia e a ultrassonografia ${ }^{(1)}$.

$\mathrm{O}$ autoexame das mamas-AEM visa estimular o autocuidado da mulher e deve ser realizado regularmente no período pós-menstrual. A sua importância se deve ao fato que no Brasil, 90\% dos casos de câncer de mama são detectados pelas próprias mulheres, o que nos leva a deduzir que a promoção do AEM seja uma estratégia eficaz para a detecção do câncer. No entanto, o INCA não estimula o AEM como estratégia isolada de detecção precoce do câncer de mama, pois as evidências científicas sugerem que o mesmo não é eficiente para o rastreamento e não contribui para a redução da mortalidade por câncer de mama. Portanto, o autoexame não substitui o exame físico realizado por profissional de saúde (médico ou enfermeiro) qualificado para essa atividade ${ }^{(1,18)}$.

Entretanto, a frequência de realização do autoexame influencia diretamente a acurácia do mesmo e estimula o autocuidado, levando as mulheres a conhecer a sua mama e a identificar alterações precocemente. Mulheres que nunca praticaram o AEM, geralmente apresentam tumores maiores, quando comparadas às que o praticam eventualmente, ou mensalmente ${ }^{(17)}$. Em nossa amostra, 65\% das mulheres pesquisadas realizam o AEM, porém apenas 2 (15,38\%) o fazem mensalmente.

O exame clínico das mamas deve ser parte do atendimento integral à mulher em todas as faixas etárias. Consiste na palpação da mama, das regiões axilares e das supraclaviculares, realizado por um profissional de saúde (médico ou enfermeiro) durante uma consulta, o qual pode detectar tumor de até um centímetro, se superficial. Recomenda-se sua realização com periodicidade anual, para as mulheres com 40 anos ou mais ${ }^{(1)}$. Neste estudo, das 13 (65\%) mulheres que realizam o exame clínico, $61,54 \%$ o fazem com frequência anual e 38,46\% esporadicamente.

A mamografia é um procedimento diagnóstico de imagem importante para a descoberta do câncer na sua fase inicial, o qual consiste em colocar a mama entre duas placas e emitir um raio-X, sendo realizado em um aparelho de alta resolução, que permite visualizar imagens tumorais e calcificações. É indicada para mulheres com suspeita e histórico de câncer acima de 35 anos, e com periodicidade de um a três anos em 
mulheres entre os 50 e 70 anos de idade ${ }^{(1)}$. Das mulheres entrevistadas, $25 \%$ tiveram o cuidado de realizar a mamografia de base, porém apenas 15\% deram seguimento em uma frequência anual.

A ultrassonografia mamária é indicada para mulheres com idade inferior a 35 anos, devido à densidade do parênquima mamário, além de permitir a diferenciação de tumores sólidos e líquidos. Na Figura 3 , observa-se que $25 \%$ das mulheres realizaram o ultrassom de base, $20 \%$ deram seguimento com frequência anual (50\%), semestral (25\%) e esporádica (25\%). Salienta-se que no município de Alfenas existem dois serviços que oferecem mamografia e ultrassonografia pelo Sistema Único de Saúde.

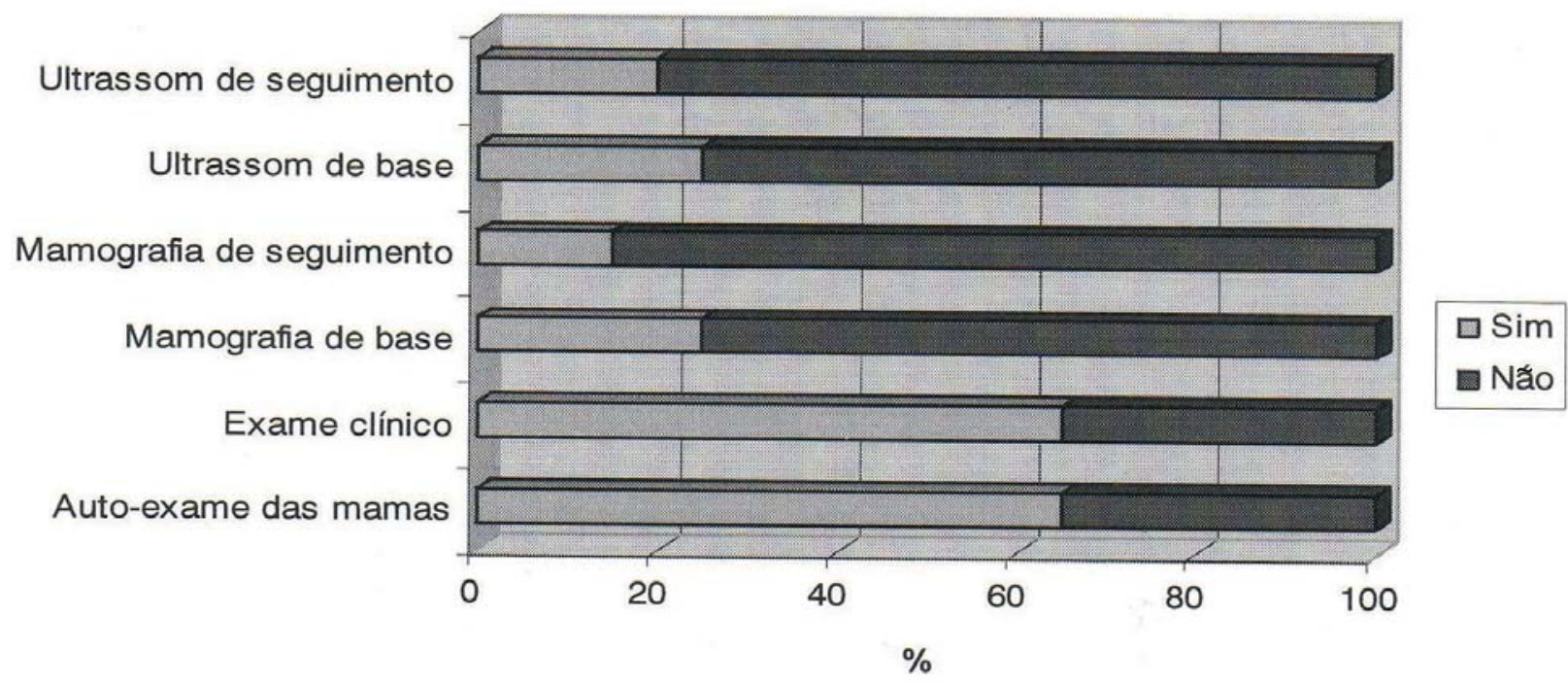

Figura 3 - Relação dos tipos de exames realizados pelas mulheres para acompanhamento da mama. Alfenas-MG, 2007

Verificou-se que essas mulheres não têm uma rotina de acompanhamento de suas mamas, tanto no que depende delas exclusivamente, que é o autoexame, como pelo acompanhamento anual, seja clínico ou por imagem.

\section{CONCLUSÃO}

Ao trabalhar com esse grupo de mulheres que tem o fator familiar para o câncer de mama, buscouse verificar se a doença de mãe ou de irmãs as levou a adotar ações de promoção e prevenção à saúde.

Verificou-se que o parentesco levou $65 \%$ dessas mulheres a realizar o exame clínico e o AEM, mas, quanto aos exames de imagem preconizado pelo INCA, apenas $25 \%$ delas o realizaram.

As participantes demonstraram desconhecimento sobre os principais fatores de risco relacionados ao câncer de mama, sendo importante que os profissionais de saúde as orientem a respeito de hábitos de vida saudáveis, como a prevenção do tabagismo, do alcoolismo, da obesidade, do sedentarismo, da importância do aleitamento materno prolongado e das práticas de detecção precoce do câncer de mama.
É imprescindível que o profissional de saúde esteja atento para perceber os limites que impedem as mulheres de dimensionar suas dificuldades em relação ao câncer de mama, bem como identificar estratégias que possibilitem às mulheres com maior risco, a adoção de práticas de autocuidado, como a realização regular do autoexame das mamas, de exame clínico e de mamografia.

\section{REFERÊNCIAS}

1. Ministério da Saúde (BR). Instituto Nacional do câncer - INCA. Programa Nacional de Controle do Câncer do Colo do Útero e de Mama - Viva Mulher. 2008 [acesso em 2008 Out 10]. Disponível : http://www.inca.gov.br.

2. Lorhisch C, Piccart M. Câncer de mama. In: Pollock et al: UICC manual de oncologia clínica. São Paulo: Fundação Oncocentro de São Paulo, 2006. Cap 23; p.505-36.

3. Freitas F, Menkei CH, Rivoire W, Passos EP. Rotina em ginecologia. Porto Alegre: Artes Médicas; 2005.

4. Jaconodino CB, Amestoy SC, Thofehrn MB. Conhecimento dos pacientes acerca dos fatores de risco 
relacionados às doenças cardiovasculares. Cogitare Enferm. 2007 Out/Dez; 12(4):466-71.

5. Rouquayrol. MZ; Almeida Filho N. Epidemiologia e saúde. Rio de Janeiro: MEDSI; 2003.

6. Cavalcanti PP. Identificando fatores de risco para câncer de mama em familiares de mulheres mastectomizadas [monografia]. Fortaleza (CE): Universidade Federal do Ceará; 2003.

7. Mcpherson K, Steel CM, Dixon JM. Breast cancer: epidemiology, risk factors and genetics. BMJ. 2000; 321:624-8.

8. Minas Gerais. $6^{\circ}$ Informativo - Vigilância do câncer e seus fatores de risco de Minas Gerais. 2008 Mar. p.12-13.

9. Colditz GA, Romer B. Cumulative risk of breast cancer to age 70 years according to risk factor status: data from the nurses health study. Am J Epidemiol. 2000 Nov; 153(10):950-62.

10. Moraes MW. Câncer de mama. In: Mohallen AGC, Rodrgues AB, organizadores. Enfermagem oncológica. São Paulo: Manole; 2007.p. 256-5.

11. Chiaradia T. Obesidade e câncer de mama: uma relação perigosa. 2007. [acesso em 2008 Out 20]. Disponível: http://dietaja.uol.com.br/Edicoes/154/artigo67009-1.asp

12. Couch FJ, Cerhan JR, Vierkant RA, Grabrick DM, Therneau TM, Pankratz VS et al. Cigarette smoking increases risk for breast cancer in high-risk breast cancer families. [periódico na Internet]. Cancer Epidemiology Biomarkers \& Prevention. 2001 [acesso em 2008 Set 05] 10:327-332. Disponível: http://research.bmn.com/ medline/search/record?biomed=yes .

13. Rossano RBA. Fatores de risco para o câncer de mama. Rev Lat-Am Mastol. 2002; 3(2).32-46

14. Thompson R. Amamentar reduz risco de câncer de mama. 2008 [acesso em 2008 Out 25]. Disponível: http:/ /www.universodamama.com.br.

15. Moura-Gallo CV, Simão TA, Ribeiro FS, Andrada-Serpa MJ, Cardoso LEB, Mendonça GAS. Mutações no gene TP53 em tumores malignos de mama: associação com fatores de risco e características clínico-patológicas, inclusive risco de óbito, em pacientes residentes no Rio de Janeiro. Rev Bras Epidemiol. 2004;7(2):167-75.

16. Godinho ER, Koch HA. Fontes utilizadas pelas mulheres para aquisição de conhecimentos sobre câncer de mama.
Radiol Bras. 2005;38(3):169-73.

17. Monteiro APS, Arraes EPP, Pontes LB, Campos MSS, Ribeiro RT, Gonçalves REB. Auto-exame das mamas: frequência do conhecimento, prática e fatores associados. Rev Bras Ginecol Obstet. 2003;25(3):201-5.

18. Batiston AP, Tamaki EM, Santos MLM,Cazola LHO. Métodos de detecção do câncer de mama e suas implicações. Cogitare Enferm. 2009 Jan/Mar;14(1)59-64. 